\title{
Intravenous immunoglobulin (IVlg) provides protection against endothelial cell dysfunction and death in ischemic stroke
}

\author{
Alexander Widiapradja ${ }^{1,2}$, Tomislav Santro ${ }^{2}$, Milan Basta ${ }^{3}$, Christopher G Sobey ${ }^{4}$, Silvia Manzanero ${ }^{2,5^{*}}$ \\ and Thiruma $\vee$ Arumugam ${ }^{1,2}$
}

\begin{abstract}
Background: The brain endothelium is a key component of the blood brain barrier which is compromised following ischemia, allowing infiltration of damaging immune cells and other inflammatory molecules into the brain. Intravenous immunoglobulin (IVIg) is known to reduce infarct size in a mouse model of experimental stroke.

Findings: Flow cytometry analysis showed that the protective effect of IVIg in ischemia and reperfusion injury in vivo is associated with reduced leukocyte infiltration, suggesting an involvement of the endothelium. In an in vitro model of ischemia, permeability analysis of the mouse brain endothelial cell line bEnd.3 revealed that IVIg prevented the loss of permeability caused by oxygen and glucose deprivation (OGD). In addition, western blot analysis of these brain endothelial cells showed that IVlg prevented the down-regulation of tight junction proteins claudin 5 and occludin and the decline in anti-apoptotic proteins BCl-2 and BCl-XL caused by OGD.
\end{abstract}

Conclusion: IVlg protects endothelial cells from ischemic insult. These studies support the use of IVIg as a pharmacological intervention for stroke therapy.

Keywords: Intravenous immunoglobulin, Ischemic stroke, Blood-brain barrier, Endothelium, Permeability

\section{Introduction}

Intravenous immunoglobulin (IVIg) is a purified concentrated human immunoglobulin solution composed primarily of IgG, obtained by fractionating blood plasma from a pool of healthy donors. Due to its immunomodulatory and anti-inflammatory effects, IVIg is used as a therapeutic modality for many immune disorders, including those that affect the nervous system [1]. We have shown that IVIg treatment reduces brain infarct volume and mortality in a mouse model of stroke [2,3]. We have also observed in in vivo and in vitro mouse models of stroke that IVIg promotes neuronal survival by inhibiting the activation of inflammasomes and apoptotic signaling and by increasing the levels of the anti-apoptotic protein B-cell lymphoma 2 (Bcl-2) [3,4].

\footnotetext{
* Correspondence: s.manzanero@uq.edu.au

${ }^{2}$ School of Biomedical Sciences, The University of Queensland, St Lucia, QLD 4072, Australia

${ }^{5}$ Australian Institute for Bioengineering \& Nanotechnology, The University of Queensland, St Lucia, QLD 4072, Australia

Full list of author information is available at the end of the article
}

In response to the local and systemic inflammation that occurs following stroke, the cerebral vasculature is subjected to endothelial cell activation, leukocyteendothelial cell adhesion, blood brain barrier (BBB) dysfunction, activation of glial cells and an enhanced generation of inflammatory mediators [5]. Clinical intervention to attenuate and/or delay $\mathrm{BBB}$ dysfunction may improve neurological outcome and facilitate patient recovery. Animal studies have shown that administration of IVIg impairs leukocyte adhesion to endothelial cells, attenuates complement-mediated damage, modulates cytokine production by various cell types and inhibits apoptosis [2,6-8]. However, it is not known whether IVIg protects from cerebrovascular dysfunction following ischemic stroke. Endothelial permeability, the ability of the endothelial cell monolayer to restrict the transfer of solutes from the blood to the brain parenchyma, is essential for cerebrovascular function. Endothelial permeability comprises paracellular and transcellular mechanisms, and tight junctions are a key component of 
the paracellular pathway, which is vulnerable to ischemic injury. Therefore, measuring tight junction proteins has been shown as a valid indicator of the paracellular pathway of endothelial permeability and its damage in ischemic injury [9]. The findings of this study reveal a beneficial effect by IVIg on ischemia-induced leukocyte recruitment as well as on endothelial permeability (mediated by tight-junction proteins claudin 5 and occludin), as well as an anti-apoptotic effect on endothelial cells mediated by Bcl-2 and Bcl-XL.

\section{Materials and methods}

\section{Focal cerebral ischemia/reperfusion (I/R) stroke model}

Three-month-old C57BL/6 J male mice were sourced from the Animal Resources Centre, Western Australia, and subjected to transient middle cerebral artery occlusion for $1 \mathrm{~h}$ followed by reperfusion, as described previously [2]. Neurological deficit, the functional abnormalities observed in mice as a result of brain damage, were scored on a five-point scale: 0 , no deficit; 1 , failure to extend right paw; 2, circling to the right; 3 , falling to the right; and 4 , unable to walk spontaneously. Only animals that showed a neurological deficit score of 1 to 3, consistent with moderate stroke damage [2,5], were used. Of 24 mice, 4 mice were excluded due to lack of neurological deficit (inclusion 83.4\%). 8 mice were administered $2 \mathrm{~g} / \mathrm{kg}$ of IVIg ( $250 \mu \mathrm{l}$; Kiovig, Baxter) by infusion into the femoral vein $3 \mathrm{~h}$ after reperfusion by an independent investigator and the remaining 12 mice were administered the vehicle phosphate-buffered saline (PBS). For each experiment, each group of mice was placed in a separate box after IVIg or vehicle injection and boxes were coded to ensure blind allocation. These experiments were approved by the Animal Care and Use Committee of The University of Queensland (Australia).

\section{Flow cytometry}

Flow cytometric analysis of immune cells was performed as previously described [5]. Animals were euthanized and perfused with PBS. Ipsilateral hemispheres were dissected, digested for $30 \mathrm{~min}$ at $37^{\circ} \mathrm{C}(1 \mathrm{mg} / \mathrm{mL}$ collagenase, $0.1 \mathrm{mg} / \mathrm{mL}$ DNAse I in Dulbecco's Modified Eagle's Medium, DMEM), and passed through a cell strainer. Cells were incubated with standard erythrocyte lysis buffer on ice and separated from myelin and debris by Percoll gradient (GE Healthcare) centrifugation. After staining of surface markers for CD45 (30-F11, 1:100) and CD11b (M170, 1:300), cells were fixed using fixation buffer (all eBioscience). Data were acquired with a LSR II FACS system and analyzed with FACSDiva (both BD Biosciences). Doublets were excluded with FSC-A and FSC-H linearity. 4 independent experiments were carried out on different days, each with 2 to 3 pooled animals per sample, to obtain adequate statistical power [5].

\section{Oxygen and glucose deprivation (OGD)}

The murine brain endothelial cell line bEnd.3 (ATCC CRL-2299) was grown to confluence in DMEM supplemented with $10 \%$ fetal bovine serum, 1\% penicillin/ streptomycin and 1\% L-glutamine (all Life Technologies). OGD was induced by exposing the cells to Locke's buffer [3] for $10 \mathrm{~min}$ before incubation in an oxygen-free chamber with $95 \% \mathrm{~N}_{2}, 5 \% \mathrm{CO}_{2}$ for 0.5-3 h. IVIg was added at the reported concentrations and proline (Sigma Aldrich), an aminoacid with no known affinity for any cell receptors, was used at a concentration of $5 \mathrm{mg} / \mathrm{mL}$ as vehicle control for all experiments.

\section{Permeability assay}

In order to assess the effect of IVIg on cell permeability during OGD, endothelial bEnd.3 cells were grown on $0.4 \mu \mathrm{m}$ pore membrane cell culture inserts (Nunc) on 24 well plates. Cells were cultured on the upper side of the insert and allowed to grow to confluence. $5 \mathrm{mg} / \mathrm{mL}$ FITC-dextran (Sigma Aldrich) was added to the upper chamber containing IVIg and Locke's buffer before exposure to OGD. After $3 \mathrm{~h}, 100 \mu \mathrm{l}$ of media from the lower chamber was collected and the amount of FITCdextran was measured using a fluorescence plate reader Varioskan (Thermo Scientific) at absorbance and emission wavelengths of $492 \mathrm{~nm}$ and $520 \mathrm{~nm}$ respectively.

\section{Lactate dehydrogenase assay}

The bEnd. 3 cells were cultured in 24 well plates until confluent before OGD treatment in the presence or absence of IVIg for $0.5,1,2$ or $3 \mathrm{~h}$. The LDH assay was performed according to the manufacturer's instructions (Roche), except that samples were incubated with the reaction solution for $1 \mathrm{~h}$. The reaction was stopped by adding $1 \mathrm{~N} \mathrm{HCl}$, and absorbance was measured on the different sample wells. Each sample measurement was carried out in triplicate.

\section{Immunoblotting}

Protein samples were run for sodium dodecyl sulfatepolyacrylamide (10\%) gel electrophoresis and then electroblotted onto a nitrocellulose membrane. The membrane was blocked for $1 \mathrm{~h}$ and incubated overnight at $4^{\circ} \mathrm{C}$ with antibodies selective for occludin (Life Technologies), claudin 5 (Life Technologies), JAM-A (Santa Cruz), ZO-1 (Abcam), AIF (Cell Signaling), Bcl-2 (Cell Signaling), Bcl-XL (Cell Signaling) and $\beta$-actin (Sigma Aldrich). After washing, the membrane was incubated with secondary antibodies for $1 \mathrm{~h}$, washed again and incubated with substrate for enhanced chemiluminescence (Pierce). After visualization on X-ray films (Fujifilm), protein levels were quantified by densitometry using Image J software. 


\section{Immunocytochemistry}

bEnd.3 cells were cultured on microscope coverslips, exposed to OGD and treated with IVIg or vehicle, and fixed in $4 \%$ paraformaldehyde. Incubation for $1 \mathrm{~h}$ with claudin 5 (Cell Signaling) antibody was followed by incubation with Alexa fluor 488 conjugated secondary antibody (Life Technologies). Nuclei were stained with DAPI and mounted with VectaShield (Vector). Images were acquired using a fluorescence microscope (Olympus).

\section{Data analysis}

Statistical power was calculated using an expected 50\% reduction in inflammatory infiltrate and permeability, and a $50 \%$ increase in protein levels between vehicle and IVIg treated samples, based on previous results from our laboratory [2-4]. All the results are reported as means \pm SEM. Overall data significance was examined by one-way analysis of variance (ANOVA). The differences between groups were considered significant at $\mathrm{p}<0.05$ using Bonferroni correction for multiple comparisons.

\section{Results}

IVIg treatment reduces leukocyte infiltration and protects against OGD-induced brain endothelial damage

Ischemic stroke promotes leukocyte recruitment to the injured tissue [5]. The first aim was to determine whether the IVIg dose previously shown as optimal to reduce infarct size $72 \mathrm{~h}$ after ischemia and reperfusion in this mouse model [2] was able to decrease leukocyte infiltration to the ischemic hemisphere at $24 \mathrm{~h}$. The chosen time point of IVIg infusion was $3 \mathrm{~h}$ post-reperfusion, as this was the latest time point previously shown to reduce infarct size [2], and late intervention supports clinical application. Our data showed that presence of CD $45^{\text {high }}$ immune cells in the ipsilateral hemisphere $24 \mathrm{~h}$ postischemia was attenuated in IVIg-treated mice compared to vehicle-treated controls (Figure $1 \mathrm{~A}$ and $\mathrm{B}$ ). Since tight regulation of endothelial permeability is pivotal to $\mathrm{BBB}$ integrity, we next assessed the effect of IVIg on permeability using the mouse brain endothelial cell line bEnd.3, measured as the ability for FITC-dextran to cross the cell monolayer $3 \mathrm{~h}$ after oxygen and glucose deprivation (OGD). IVIg-treated cells exhibited reduced permeability compared with control cells (Figure 1C). In order to investigate whether this was a consequence of tight junction protein modulation, we assessed expression levels of claudin 5, occludin, junctional adhesion molecule 1 (JAM-1) and zona occludens-1 (ZO-1). Immunoblot analyses showed that expression of each of these tight junction proteins was reduced after 3 h OGD (Figure 1D$\mathrm{H})$. However, the decline in claudin 5 and occludin levels was completely rescued in the presence of IVIg (Figure 1E and F). Immunocytochemistry confirmed the disappearance of claudin 5 from the membranes of OGD-treated endothelial cells and the conservation of claudin 5 integrity in the presence of IVIg (Figure 1I). Overall these results point to a beneficial effect of IVIg on the maintenance of tight-junction proteins claudin 5 and occludin during ischemic stress, which would strengthen the BBB and contribute to a decreased leukocyte infiltration after ischemia.

\section{IVlg protects brain endothelial cells against OGD-induced} cell death by up-regulating anti-apoptotic proteins

Next, we investigated the effect of IVIg on the survival of brain endothelial cells during OGD. bEnd.3 cells treated with IVIg showed consistently low levels of LDH release throughout an OGD time course up to $3 \mathrm{~h}$, indicative of a protective effect of IVIg against OGD-induced cell death (Figure 2A). In order to better understand the underlying mechanisms, analyses of apoptosis inducing factor (AIF), Bcl-2, and Bcl-XL were carried out by immunoblotting. IVIg treatment tended to reduce the increase in AIF observed following 2-3 $\mathrm{h}$ OGD, however this effect did not reach statistical significance (Figure 2B and 2C). However, IVIg significantly prevented the OGD-induced decreases of both $\mathrm{Bcl}-2$ and Bcl-XL observed in untreated cultures (Figure 2D-F). These data support an action of IVIg to protect cerebral endothelial cells from undergoing ischemia-induced apoptosis.

\section{Discussion}

We $[2,3]$ and others $[10,11]$ have previously demonstrated that IVIg treatment reduces brain infarct volume and mortality in experimental rodent models of stroke. This study reveals a protective effect of IVIg against endothelial dysfunction following ischemic stroke, in association with reduced leukocyte infiltration in vivo. The data indicate that IVIg provides a two-way protective mechanism for cerebral endothelial cells, firstly by blocking the increase in BBB permeability involving downregulation of tight-junction proteins claudin 5 and occludin, and secondly by preventing ischemia-induced endothelial cell death involving a reduction in anti-apoptotic proteins, $\mathrm{Bcl}-2$ and Bcl-XL.

This study shows for the first time that infusion of a high dose of IVIg ( $2 \mathrm{~g} / \mathrm{kg}$ body weight) $3 \mathrm{~h}$ after reperfusion reduces leukocyte infiltration into the brain. Ischemic stroke causes endothelial cell dysfunction with the breakdown of tight junction proteins and compromised BBB function, leading to increased protein extravasation, interstitial edema and the adhesion and transmigration of leukocytes [12]. We have previously demonstrated the injurious role of infiltrating immune cells in the pathogenesis of post-ischemic brain injury in mice [5], and the beneficial effects of IVIg treatment on the levels of inflammatory endothelial and leukocyte adhesion molecules 
A

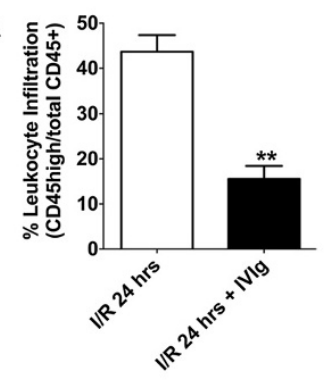

C

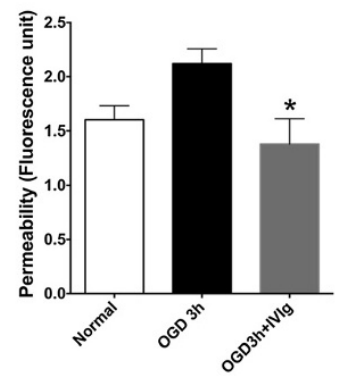

E

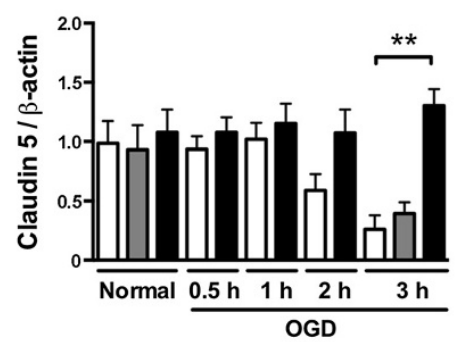

G

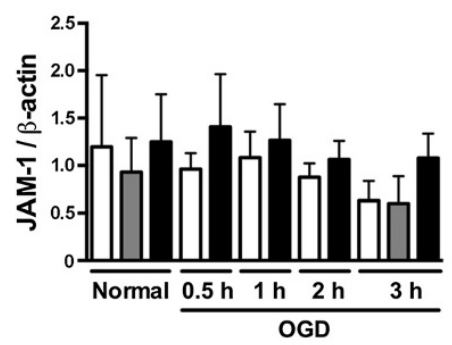

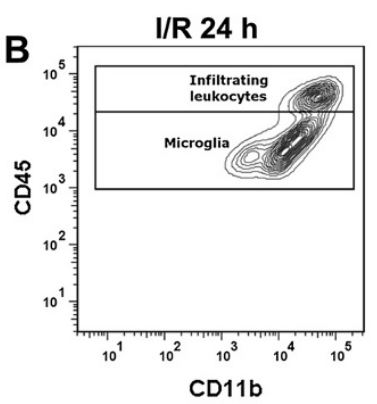

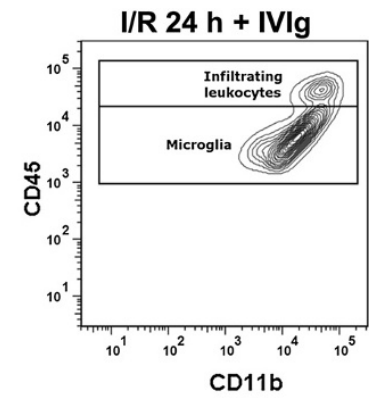

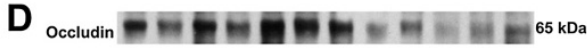
Claudin $5=-\omega-6-0 \quad-\quad-22 \mathrm{kDa}$

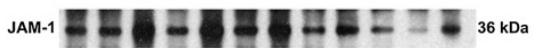

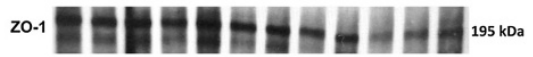
Actin -

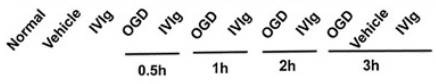
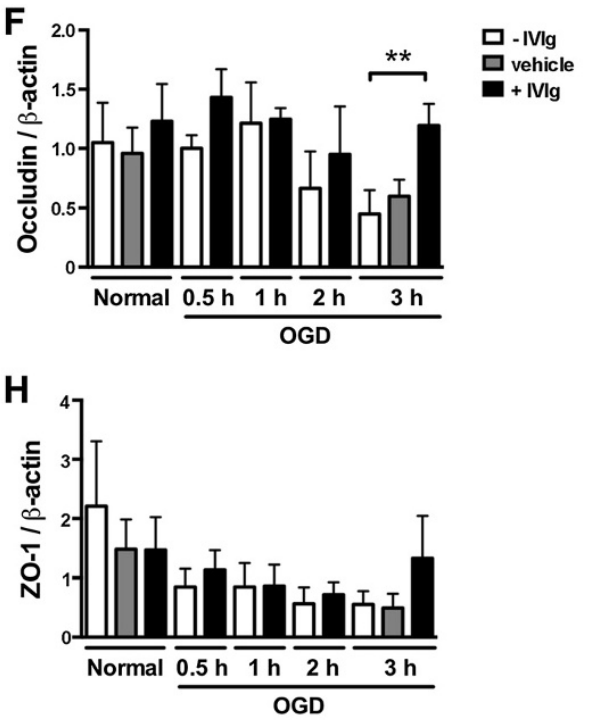

I Claudin 5 / DAPI

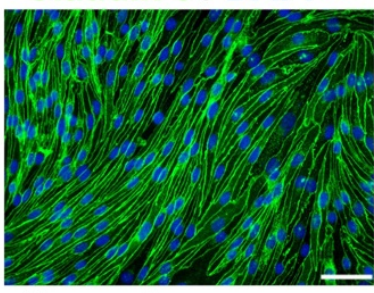

Normal

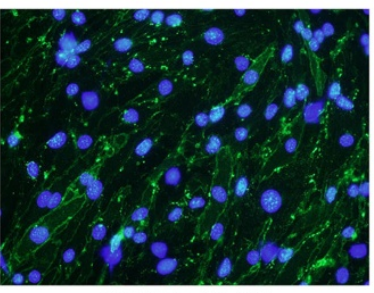

OGD (3 h)

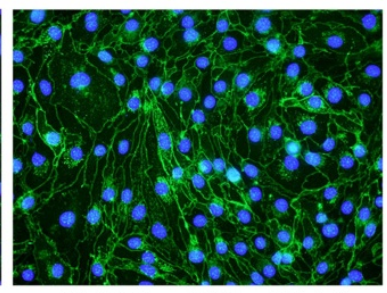

OGD + IVIg (3 h)

Figure 1 IVlg prevents post-ischemic leukocyte infiltration and maintains blood brain barrier integrity. (A) Administration of IVlg (2 g/kg) decreased leukocyte infiltration following $1 \mathrm{~h}$ and $23 \mathrm{~h}$ respectively of cerebral ischemia and reperfusion (I/R). ${ }^{* *} \mathrm{p}<0.01$ compared to $\mathrm{l} / \mathrm{R}$ mice, $\mathrm{n}=8-12$. (B) Representative flow cytometry plots showing the two studied populations of CD45 cells, infiltrating leukocytes (CD45 high) and microglia (CD45 intermediate). (C) Treatment with high concentration of $\mathrm{IVIg}(5 \mathrm{mg} / \mathrm{mL})$ reduced permeability of bEnd.3 cell monolayers subjected to oxygen and glucose deprivation (OGD). ${ }^{*} p<0.05$ compared to OGD 3 h. (D, E and F) IVlg rescues the decrease in claudin 5 and occludin levels that occurs after $3 \mathrm{~h}$ OGD in untreated or vehicle controls. ${ }^{* *} \mathrm{p}<0.01$. (G and H) A similar trend was seen in the expression levels of JAM-1 and ZO-1, however statistical significance was not reached. (I) Immunofluorescence staining showed the restoration of claudin 5 expression in IVlg-treated bEnd.3 cells subjected to 3 h OGD. Scale bar: $20 \mu \mathrm{m}$. 

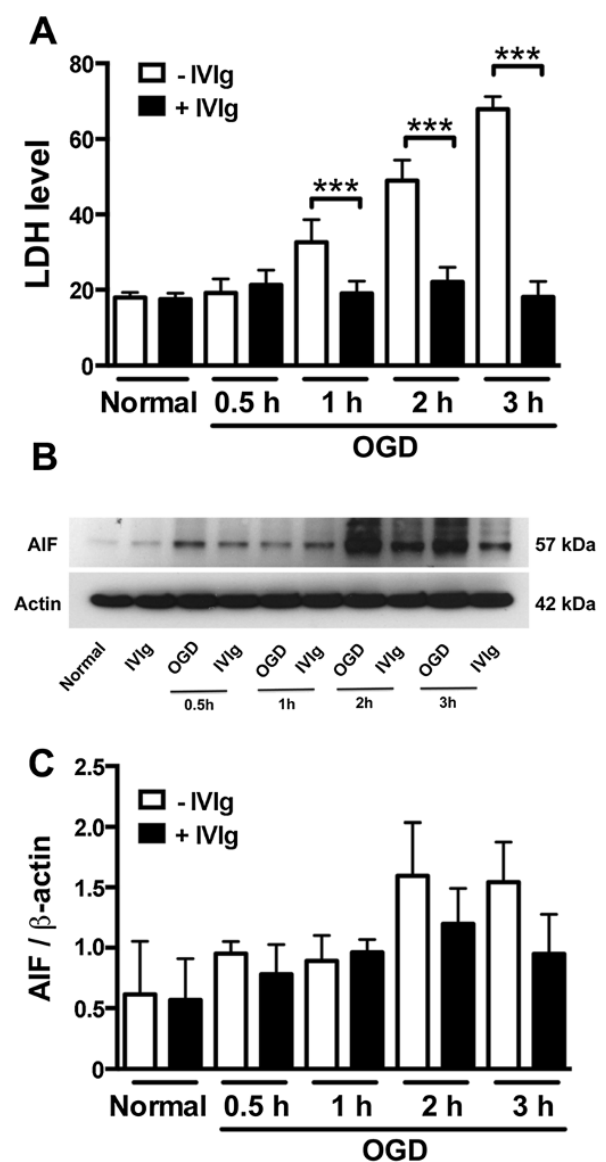

D
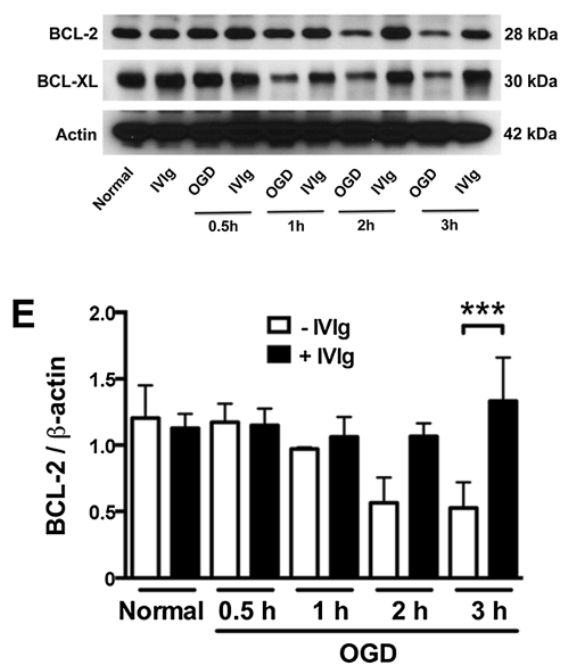

$\mathbf{F}$

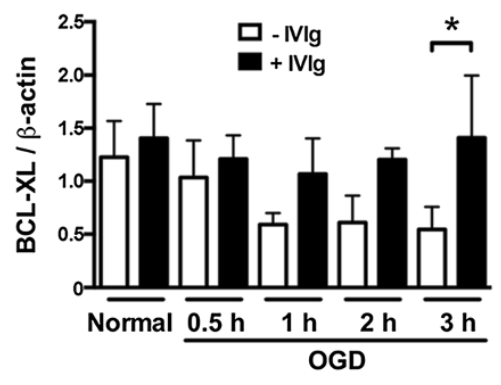

Figure 2 IVIg promotes endothelial cell survival in OGD. (A) IVlg treatment reduced LDH release from bEnd.3 cells subjected to 1-3 h OGD. ${ }^{* * *} \mathrm{p}<0.001$ as indicated by bars. (B and C) IVIg moderated the increase in AlF levels in bEnd.3 cells, however statistical significance was not reached. (D, E and F) IVIg prevented the decrease in BCl-2 and Bcl-XL levels measured in bEnd.3 lysates following $3 \mathrm{~h} \mathrm{OGD.}{ }^{* * *} \mathrm{p}<0.001,{ }^{*} p<0.05$ as indicated by bars.

such as ICAM-1, CD11a, and CD11b in a mouse model of ischemic stroke [2].

Ischemic stroke compromises $\mathrm{BBB}$ integrity, which is mediated by cytoskeleton rearrangement, and redistribution and disappearance of tight junction proteins such as claudin 5 and occludin in brain endothelial cells, resulting in increased $\mathrm{BBB}$ permeability [13]. Our results show that endothelial permeability, which is vulnerable to OGD, remains unaffected by OGD in the presence of IVIg. In accordance with this finding, the loss of key tight-junction proteins claudin 5 and occludin in OGD does not occur when IVIg is present. IVIg could, therefore, prevent the loss of tight junction integrity in the brain and consequently protect the $\mathrm{BBB}$ whose breakdown contributes to a large part of stroke-induced injury. A recent study investigating the effect of IVIg on BBB integrity in a sepsis model suggested that immunoglobulin $\mathrm{G}, \mathrm{A}$, or $\mathrm{M}$ improved the integrity of the BBB and inhibited symptoms of sickness in rats [14]. We previously reported that IVIg crosses the $\mathrm{BBB}$ and exerts protective effects in a mouse model of stroke [2,3]. There are two ways in which IVIg may be able to reduce BBB permeability and at the same time be able to cross the $\mathrm{BBB}$ - while IVIg may cross the intact $\mathrm{BBB}$, as reported recently in mouse [15], it is also possible that IVIg crosses the damaged BBB and then exerts its permeability modulation once in the brain parenchyma.

This study highlights a previously unrecognised beneficial action of IVIg on cerebral endothelial cells exposed to ischemia, in that cell death is inhibited and levels of $\mathrm{Bcl}-2$ and $\mathrm{Bcl}-\mathrm{XL}$ are preserved. Bcl-2 and $\mathrm{Bcl}-\mathrm{XL}$ play pivotal roles in determining cell survival or death under conditions of stress, and have both been shown to prevent endothelial cell death [16]. Ischemic conditions, or exposure to $A \beta$, promote a decline in Bcl-2 levels and subsequently increase apoptosis in mouse primary cortical neurons, but the presence of IVIg maintains high $\mathrm{Bcl}-2$ levels and low cell death rates [3]. In physiological terms, IVIg could potentially be responsible for the survival of a number of vessels in the penumbra that would otherwise 
undergo cell death from ischemia, hence contributing to the extension of the infarct. Taken together, these data indicate that IVIg has significant protective characteristics for inhibiting endothelial, as well as neuronal cell death following ischemic stroke. In addition to the known neuroprotective role of IVIg, we have demonstrated that IVIg acts through multiple mechanisms to protect brain tissue after stroke. Specifically, our present findings provide evidence that IVIg inhibits endothelial dysfunction and death and reduces leukocyte infiltration following ischemic stroke.

The current view in stroke treatment supports the notion of stroke therapies that affect multiple injury mechanisms simultaneously. IVIg, with its effects on neurons, inflammatory cells and endothelium fits this definition perfectly and as such promises to be a relevant candidate for translational research to the clinic.

\section{Competing interests}

The authors declare that they have no competing interests.

\section{Authors' contributions}

AW, CGS, MB and TVA conceived the study; TVA and SM coordinated the study; AW carried out protein expression studies and cell death assays with TS help; AW and TS carried out permeability assays; SM, AW, TS and TVA carried out flow cytometry; AW, SM, TVA, CS and MB wrote the manuscript. All authors read and approved the final manuscript.

\section{Acknowledgements}

This research was supported by the Australian Research Council Future Fellowship to TVA (ARCFT100100427) and the start-up fund provided by the National University of Singapore. CGS is a Senior Research Fellow of the National Health and Medical Research Council of Australia.

\section{Author details}

'Department of Physiology, Yong Loo Lin School of Medicine, National University of Singapore, Singapore 117597, Singapore. ${ }^{2}$ School of Biomedical Sciences, The University of Queensland, St Lucia, QLD 4072, Australia. ${ }^{3}$ Biovisions, Inc., Potomac, MD, USA. ${ }^{4}$ Department of Pharmacology, Monash University, Clayton, Victoria, Australia. ${ }^{5}$ Australian Institute for Bioengineering \& Nanotechnology, The University of Queensland, St Lucia, QLD 4072, Australia.

Received: 9 April 2014 Accepted: 6 June 2014

Published: 20 June 2014

\section{References}

1. McCormack PL: Immune globulin (human) $10 \%$ liquid: a review of its use in primary immunodeficiency disorders. BioDrugs 2013, 27:393-400.

2. Arumugam TV, Tang SC, Lathia JD, Cheng A, Mughal MR, Chigurupati S, Magnus T, Chan SL, Jo DG, Ouyang X, Fairlie DP, Granger DN, Vortmeyer A, Basta M, Mattson MP: Intravenous immunoglobulin (IVIG) protects the brain against experimental stroke by preventing complement-mediated neuronal cell death. Proc Natl Acad Sci U S A 2007, 104:14104-14109.

3. Widiapradja A, Vegh V, Lok KZ, Manzanero S, Thundyil J, Gelderblom M, Cheng YL, Pavlovski D, Tang SC, Jo DG, Magnus T, Chan SL, Sobey CG, Reutens D, Basta M, Mattson MP, Arumugam TV: Intravenous immunoglobulin protects neurons against amyloid beta-peptide toxicity and ischemic stroke by attenuating multiple cell death pathways. J Neurochem 2012, 122:321-332.

4. Fann DY, Lee SY, Manzanero S, Tang SC, Gelderblom M, Chunduri P, Bernreuther C, Glatzel M, Cheng YL, Thundyil J, Widiapradja A, Lok KZ, Foo SL, Wang YC, Li YI, Drummond GR, Basta M, Magnus T, Jo DG, Mattson MP, Sobey CG, Arumugam TV: Intravenous immunoglobulin suppresses NLRP1 and NLRP3 inflammasome-mediated neuronal death in ischemic stroke. Cell Death Dis 2013, 4:e790.

5. Gelderblom M, Leypoldt F, Steinbach K, Behrens D, Choe CU, Siler DA, Arumugam TV, Orthey E, Gerloff C, Tolosa E, Magnus T: Temporal and spatial dynamics of cerebral immune cell accumulation in stroke. Stroke 2009, 40:1849-1857.

6. Issekutz AC, Rowter D, Macmillan HF: Intravenous immunoglobulin G (IVIG) inhibits IL-1- and TNF-a-dependent, but not chemotactic-factor-stimulated, neutrophil transendothelial migration. Clin Immunol 2011, 141:187-196.

7. Jang JE, Hidalgo A, Frenette PS: Intravenous immunoglobulins modulate neutrophil activation and vascular injury through FcyRIII and SHP-1. Circ Res 2012, 110:1057-1066.

8. Basta M: Ambivalent effect of immunoglobulins on the complement system: activation versus inhibition. Mol Immunol 2008, 45:4073-4079.

9. Sandoval KE, Witt KA: Blood-brain barrier tight junction permeability and ischemic stroke. Neurobiol Dis 2008, 32:200-219.

10. Tunik S, Aluclu MU, Acar A, Akkoc H, Guzel A, Alabalik U, Akkus M: The effects of intravenous immunoglobulin on cerebral ischemia in rats: an experimental study. Toxicol Ind Health 2013, [Epub ahead of print]

11. Walberer M, Nedelmann M, Ritschel N, Mueller C, Tschernatsch M, Stolz E, Bachmann G, Blaes F, Gerriets T: Intravenous immunoglobulin reduces infarct volume but not edema formation in acute stroke. Neuroimmunomodulation 2010, 17:97-102.

12. Rodrigues SF, Granger DN: Leukocyte-mediated tissue injury in ischemic stroke. Curr Med Chem 2014, 21:2130-2137.

13. Fernández-López D, Faustino J, Daneman R, Zhou L, Lee SY, Derugin N, Wendland MF, Vexler ZS: Blood-brain barrier permeability is increased after acute adult stroke but not neonatal stroke in the rat. $J$ Neurosci 2012, 32:9588-9600.

14. Esen F, Senturk E, Ozcan PE, Ahishali B, Arican N, Orhan N, Ekizoglu O, Kucuk $M$, Kaya M: Intravenous immunoglobulins prevent the breakdown of the blood-brain barrier in experimentally induced sepsis. Crit Care Med 2012, 40:1214-1220.

15. St-Amour I, Paré I, Alata W, Coulombe K, Ringuette-Goulet C, Drouin-Ouellet J, Vandal M, Soulet D, Bazin R, Calon F: Brain bioavailability of human intravenous immunoglobulin and its transport through the murine blood-brain barrier. J Cereb Blood Flow Metab 2013, 33:1983-1992.

16. Sawant DA, Tharakan B, Wilson RL, Stagg HW, Hunter FA, Childs EW: Regulation of tumor necrosis factor-a-induced microvascular endothelial cell hyperpermeability by recombinant B-cell lymphoma-extra large. J Surg Res 2013, 184:628-637.

doi:10.1186/2040-7378-6-7

Cite this article as: Widiapradja et al.: Intravenous immunoglobulin (IVIg) provides protection against endothelial cell dysfunction and death in ischemic stroke. Experimental \& Translational Stroke Medicine 2014 6:7.

\section{Submit your next manuscript to BioMed Central and take full advantage of:}

- Convenient online submission

- Thorough peer review

- No space constraints or color figure charges

- Immediate publication on acceptance

- Inclusion in PubMed, CAS, Scopus and Google Scholar

- Research which is freely available for redistribution 